\title{
Wide range quantitative photoacoustic spectroscopy to measure non-linear optical absorption of hemoglobin
}

Amos Danielli, Konstantin Maslov, Jun Xia, Lihong V. Wang

Amos Danielli, Konstantin Maslov, Jun Xia, Lihong V. Wang, "Wide range quantitative photoacoustic spectroscopy to measure non-linear optical absorption of hemoglobin," Proc. SPIE 8223, Photons Plus Ultrasound: Imaging and Sensing 2012, 82233H (23 February 2012); doi: $10.1117 / 12.908900$

SPIE. Event: SPIE BiOS, 2012, San Francisco, California, United States 


\title{
Wide range quantitative photoacoustic spectroscopy to measure non- linear optical absorption of hemoglobin
}

\author{
Amos Danielli ${ }^{\dagger}$, Konstantin Maslov ${ }^{\dagger}$, Jun Xia, Lihong V. Wang ${ }^{*}$ \\ Optical Imaging Laboratory, Department of Biomedical Engineering, Washington University in St. \\ Louis, MO 63130 \\ ${ }^{\dagger}$ These authors contributed equally to this work
}

\begin{abstract}
Photoacoustic microscopy (PAM) has been shown to be a valuable tool for quantifying hemoglobin oxygenation within single vessels. Recently, optical-resolution PAM was developed to achieve higher resolution by reducing the laser beam diameter, which increased the light intensity. As intensity increases, saturation of the optical absorption and multiphoton/multi-step absorption can occur, which, together with the temperature dependence of thermal expansion, result in a non-linear dependence of the photoacoustic signal on the excitation pulse fluence. For hemoglobin, the major absorber in tissue for photoacoustic imaging, these non-linear phenomena have strong wavelength dependence. To enable quantitative photoacoustic measurements at different wavelengths in the presence of nonlinearity, a careful wide range analysis of the intensity-dependent absorption is required. Here, we built a photoacoustic spectrometer, using a tunable nanosecond optical parametric oscillator that operates between $410 \mathrm{~nm}$ and $2400 \mathrm{~nm}$ as our light source. To reduce uncertainty in our measurements due to inhomogeneous spatial distribution of the optical fluence, we used a flat-top beam illumination and a flat transducer which was mounted in reflection mode, effectively reducing quantitative measurements to a one dimensional problem. Intensity-dependent non-linear spectra of the photoacoustic signals of oxyand deoxy-hemoglobin were obtained. These measurements have the potential to contribute significantly to quantitative functional PAM.
\end{abstract}

Keywords: Photoacoustic microscopy; non-linear photoacoustic microscopy; oxygen saturation; optical saturation; photoacoustic tomography; OR-PAM

\section{INTRODUCTION}

\subsection{Quantitative photoacoustic microscopy}

Photoacoustic microscopy is an effective functional and molecular imaging tool in vivo. Because the photoacoustic (PA) signal is proportional to the deposited optical energy, which in turn is proportional to the local optical absorption coefficient, multi-wavelength photoacoustic measurements provide quantitative information about the concentrations of multiple chromophores, such as oxygenated and deoxygenated hemoglobin molecules in red blood cells. Thus, the relative concentration and the oxygen saturation $\left(\mathrm{sO}_{2}\right)$ of hemoglobin can be extracted ${ }^{1-3}$. All quantitative functional photoacoustic studies have assumed a linear dependence between the photoacoustic signal and the local optical fluence, and used the linear least squares model. Recently, we showed that non-linear effects, such as saturation of the optical absorption, thermal dependence of the Grueneisen coefficient, and/or multi-photon/multi-step excitation affect the quantitative measurements both in vitro and in vivo ${ }^{4}$. For hemoglobin, the major absorber in tissue for photoacoustic imaging, these non-linear phenomena have strong wavelength dependence. The objective of this paper is to demonstrate the intensity-dependent non-linear spectra of the PA signals of oxy- and deoxy-hemoglobin and to identify wavelengths most beneficial for quantitative PAM.

\subsection{Non-linear photoacoustic microscopy}

In an absorbing medium, the photoacoustic (PA) signal, q, generated by single photon absorption is assumed to be proportional to the local pressure rise ${ }^{5}$ :

$$
q(F) \propto \Gamma \cdot \eta_{t h} \cdot \mu_{a} \cdot F
$$

*1hwang@biomed.wustl.edu

Photons Plus Ultrasound: Imaging and Sensing 2012, edited by Alexander A. Oraevsky, Lihong V. Wang, Proc. of SPIE Vol. 8223, 82233H - @ 2012 SPIE · CCC code: 1605-7422/12/\$18 - doi: 10.1117/12.908900 
where $\Gamma$ is the Grueneisen parameter (dimensionless), $\eta_{t h}$ is the percentage of absorbed energy that is converted to heat, $\mu_{\mathrm{a}}$ is the optical absorption coefficient $\left(\mathrm{cm}^{-1}\right)$, and $F$ is the local optical fluence $\left(\mathrm{J} / \mathrm{cm}^{2}\right)$. The local optical fluence can also be written as $F=I \cdot \tau_{\text {laser }}$, where $I$ is the local laser intensity $\left(\mathrm{W} / \mathrm{cm}^{2}\right)$ and $\tau_{\text {laser }}$ is the laser pulse width. At low intensity, the absorption coefficient can be written as the absorption cross section, $\sigma_{a}$, multiplied by the number of absorbers per unit volume, $N_{0}$ :

$$
\mu_{a}=\sigma_{a} \cdot N_{0},
$$

As intensity increases, mechanisms such as saturation of the optical absorption or multi-photon/multi-step absorption can occur, resulting in a non-linear dependence of the photoacoustic signal on the excitation pulse fluence ${ }^{6-8}$

$$
q(F) \propto \Gamma \cdot \eta_{t h} \cdot \sigma_{a} \cdot \frac{N_{0}}{1+\frac{I}{I_{s a t}}} \cdot F+k_{1} \cdot F^{2},
$$

where $I_{\text {sat }}$ is the saturation intensity and $k_{l}$ is the two-photon / multi-step absorption coefficient. The saturation intensity is at minimum when the absorption cross section is at maximum. For example, for oxyhemoglobin, the photoacoustic signal saturates at wavelengths near the absorption peaks at $578 \mathrm{~nm}$ and $540 \mathrm{~nm}$ (see Figure 1).

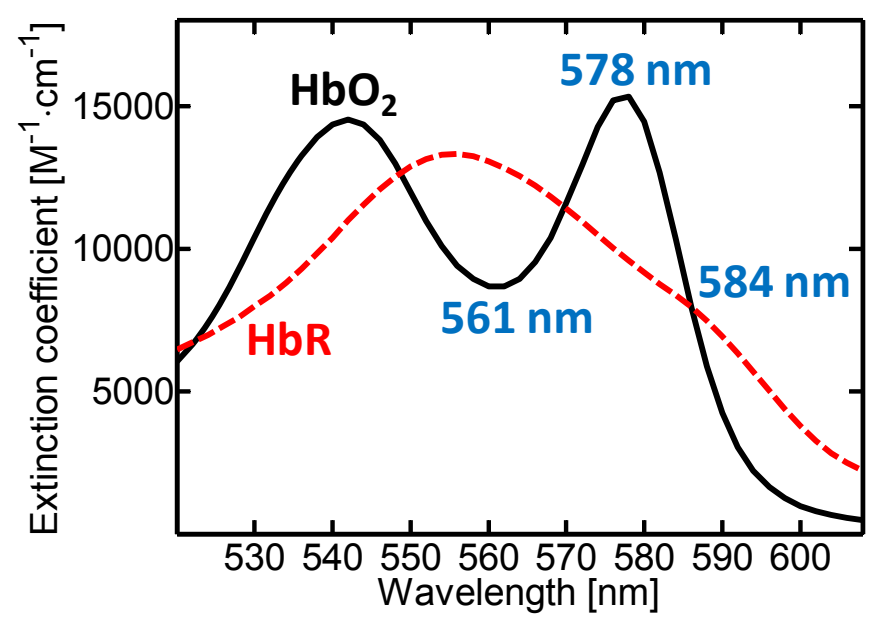

Figure 1: Molar extinction coefficient of oxyhemoglobin (black line) and deoxyhemoglobin (dashed red line).

Moreover, when the temperature rise due to the absorption is high, the Grueneisen parameter, which depends on the temperature, changes':

$$
\begin{aligned}
& \Gamma(T) \cong \Gamma\left(\mathrm{T}_{0}\right)+\frac{\partial \Gamma}{\partial T} \cdot \Delta T=\Gamma_{0}+\mathrm{k}_{2} \cdot \mu_{a} \cdot F, \\
& \Delta T \propto \mu_{a} \cdot F
\end{aligned}
$$

where $k_{2}$ is a linear approximation coefficient of the Grueneisen parameter. The resulted photoacoustic signal is

$$
q(F) \propto\left(\Gamma_{0}+\mathrm{k}_{2} \cdot \mu_{a} \cdot F\right) \cdot \eta_{t h} \cdot \mu_{a} \cdot F=\Gamma_{0} \cdot \eta_{t h} \cdot \mu_{a} \cdot F+\mathrm{k}_{2} \cdot \eta_{t h} \cdot\left(\mu_{a} \cdot F\right)^{2} .
$$

\section{EXPERIMENTAL SETUP AND RESULTS}

\subsection{Flat-top PAM}

We measured the photoacoustic signal as a function of the incident laser fluence, using a custom-built PAM system with a flat top beam illumination shown in Figure 2. A Q-Switched Nd:YAG laser (Quantel Inc.) with $3^{\text {rd }}$ harmonic $(355 \mathrm{~nm})$ output was used to pump an OPO laser (BasiScan, Newport), which provided lasing at $10 \mathrm{~Hz}$ in the wavelength range of 410-1050 $\mathrm{nm}$. The ultrasonic and optic coupling cube was made of a BK7 glass prism joined with rhomboidal prism with a layer of index matching liquid between them. The liquid layer allows light beam to pass freely towards a sample cell, while reflecting the ultrasound pulse coming from the sample. Ultrasonic reflection occurs with near $100 \%$ 
conversion into a shear wave, which after second reflection from a free surface, is converted back to longitudinal wave and detected by an attached $30 \mathrm{MHz}$ ultrasonic transducer (V213-BC, Olympus). A $3 \mathrm{~mm}$ thick Teflon sample cell with $3 \mathrm{~mm}$ inner diameter was attached to the prism. Several micro-liters of dye solution of interest were deposited into the cell on the prism surface. During the experiments, the cell was fully filled with solution to decrease the effect of photobleaching and to assure constant concentration of the dye. However, equivalent thickness of the cell is roughly equal to the ultrasonic wavelength in solvent, which is approximately $50 \mu \mathrm{m}$. Hence, minimal amount of solution can be as small as $50 \mathrm{nl}$. To measure the laser pulse energy, a small portion of the light was reflected to a pyroelectric detector (SPH-11, Spectrum Detector Inc.), which was calibrated for a wide range of laser pulse energies over the entire wavelength range. The signals from the ultrasonic transducer and the pyroelectric detector were digitized using a 14 bit, $200 \mathrm{MHz}$ digitizer (14200, Gage). To minimize non-linear dependence of the photoacoustic signal on the beam profile, the laser beam profile was kept equally constant across the area of illumination using an engineered diffuser (PRC Photonics) that was incorporated into the cell design. This diffuser provided 5-10\% uniformity within a $1 \mathrm{~mm}$ optical beam diameter at the sample cell surface (see Figure 2). The diffuser had a high laser damage threshold, and a broad transmission spectrum spanning from $180-2500 \mathrm{~nm}$.
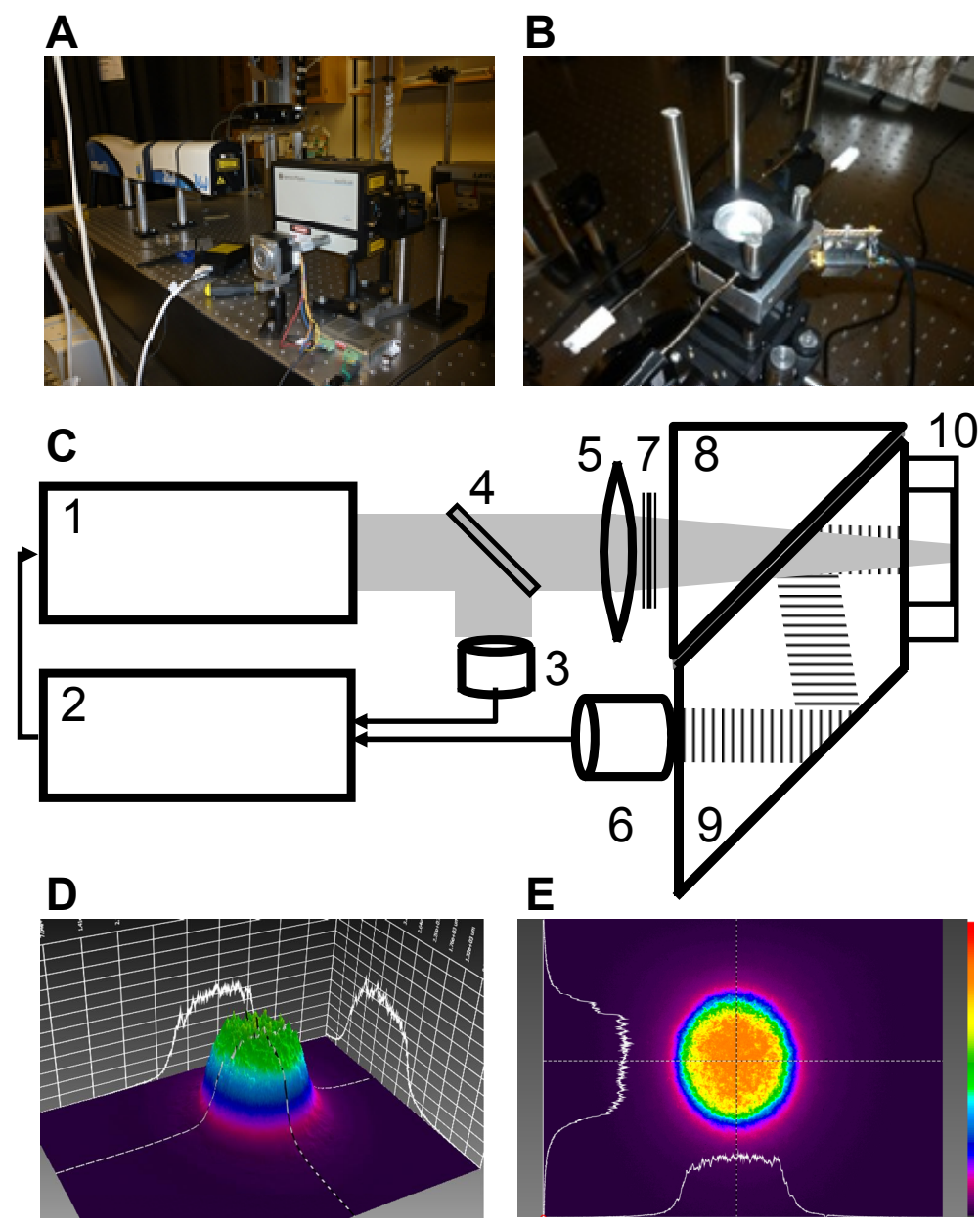

Figure 2: PAM system with a flat top beam illumination. A. Photoacoustic spectrophotometer, B. photoacoustic cell, C. Schematic of the photoacoustic spectrophotometer: 1. Tunable pulse laser, 2. Data acquisition system, 3 Pyroelectric sensor, 4. Beam sampler, 5. Focusing lens, 6. Ultrasonic transducer, 7. Engineered diffuser, 8. Right angle prism,.9. Rhomboidal prism, 10 Dye cell, D. Beam profile (3D), E. Beam profile (2D).

\subsection{Oxygenated hemoglobin}

We measured the photoacoustic signal (maximum peak to peak amplitude) from $\mathrm{HbO}_{2}$ and $\mathrm{HbR}$ as a function of the incident pulse energy in the wavelength range $450-650 \mathrm{~nm}$. The PA signal at each wavelength was averaged using 20 
pulses and normalized by the averaged pulse energy. The pulse repetition rate was $10 \mathrm{~Hz}$. Oxygenated lysed bovine blood (50:50 DI-water and defibrinated bovine blood) was purchased from Quadfive (\#905-250) and further diluted (1:10) with DI-water. The oxygenated level was measured to be $100 \%$ using a spectrophotometer.
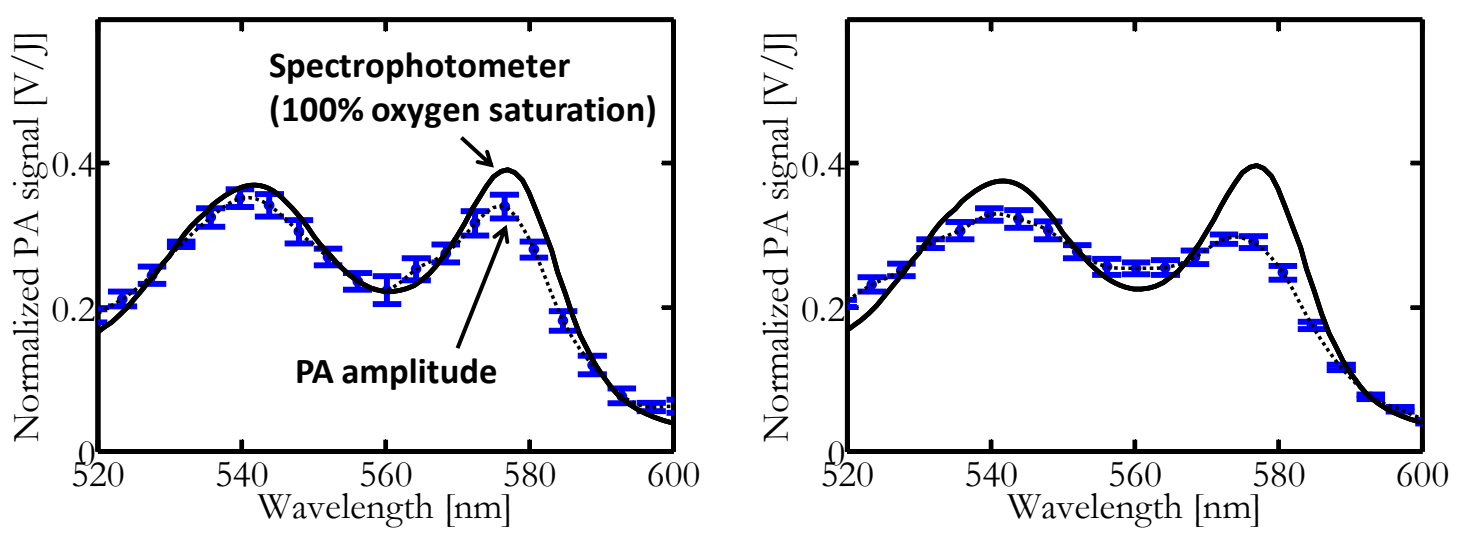

Figure 3: The PA amplitude as a function of the wavelength in fully oxygenated hemoglobin at (a) Low fluence, and (b) high fluence. The solid line is the measured absorption using a spectrophotometer and it fits the theoretical absorption of $100 \%$ oxygenated hemoglobin.

The PA amplitude as a function of the incident fluence is presented in the following figures for different wavelengths.
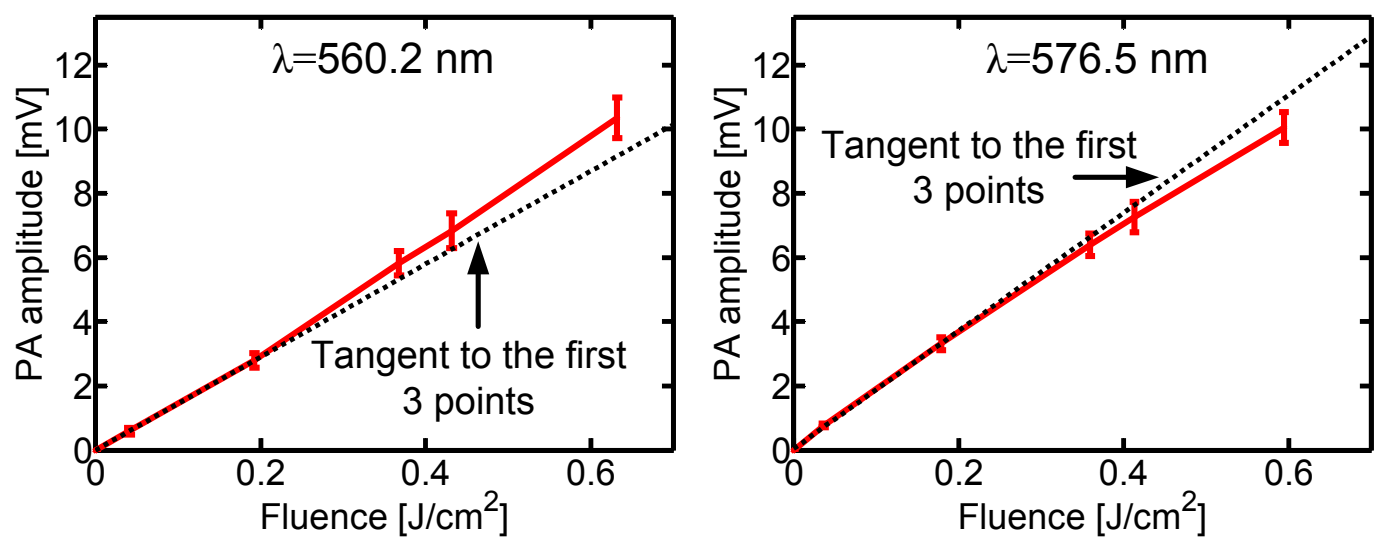

Figure 4: The PA amplitude as a function of the incident fluence at different wavelengths.

At the absorption peaks (576 nm and $540 \mathrm{~nm}$ ) of oxy-hemoglobin, saturation is the dominant non-linear phenomenon. At other wavelengths (e.g., $560 \mathrm{~nm}$ ), the dominant non-linear phenomenon has quadratic dependent, suggesting either thermal non-linearity or multi-step excitation.

\subsection{Deoxygenated hemoglobin}

Deoxygneated blood was measured in a dead mouse tissue. The mouse was euthanized by putting it into a chamber of $100 \%$ carbon dioxide, which induced hypoxemia. After confirming death, the mouse was put in a fridge for one hour. The scalp and skull were then removed, and a thin slice of brain tissue was excised. The tissue was mainly gray matter, which is rich in capillary beds. 

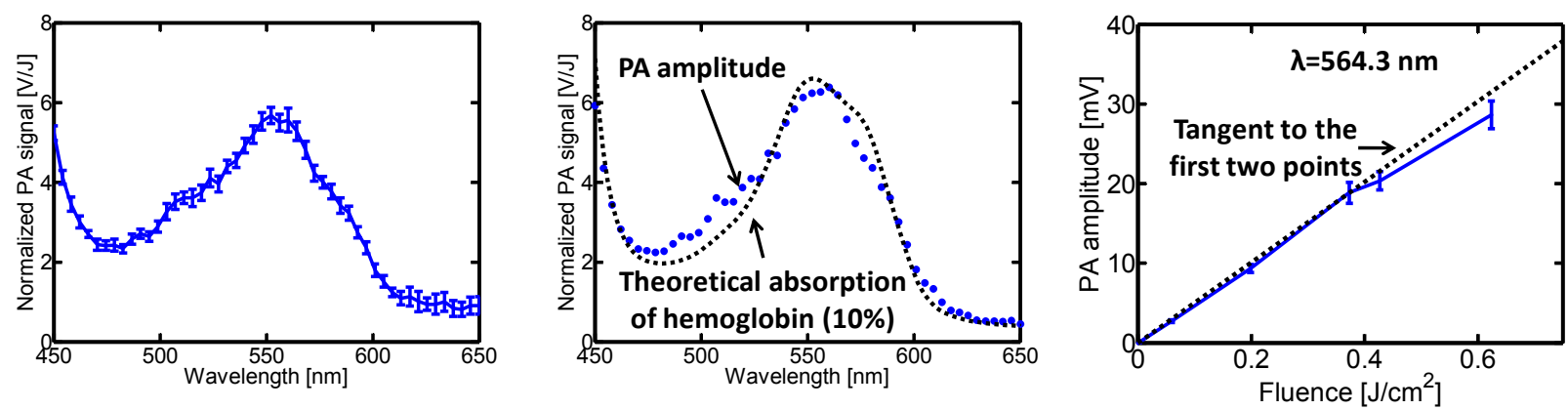

Figure 5: (a) The PA amplitude as a function of the wavelength in deoxygenated hemoglobin (b) The fitted theoretical absorption of $10 \%$ oxygen saturated hemoglobin (c) The PA amplitude as a function of the incident fluence at $564.3 \mathrm{~nm}$

\subsection{Wavelength dependent quadratic coefficient}

To evaluate the amount and type of non-linearity, at each wavelength we fitted the photoacoustic amplitude as a function of the fluence to a second order polynomial function:

$$
P A=a_{1} \cdot F+a_{2} \cdot F^{2}
$$

When the quadratic coefficient is negative, the dominant non-linear effect at the corresponding wavelength is saturation. When the quadratic coefficient is positive, the dominant non-linear effect is thermal non-linearity or multi-step excitation.
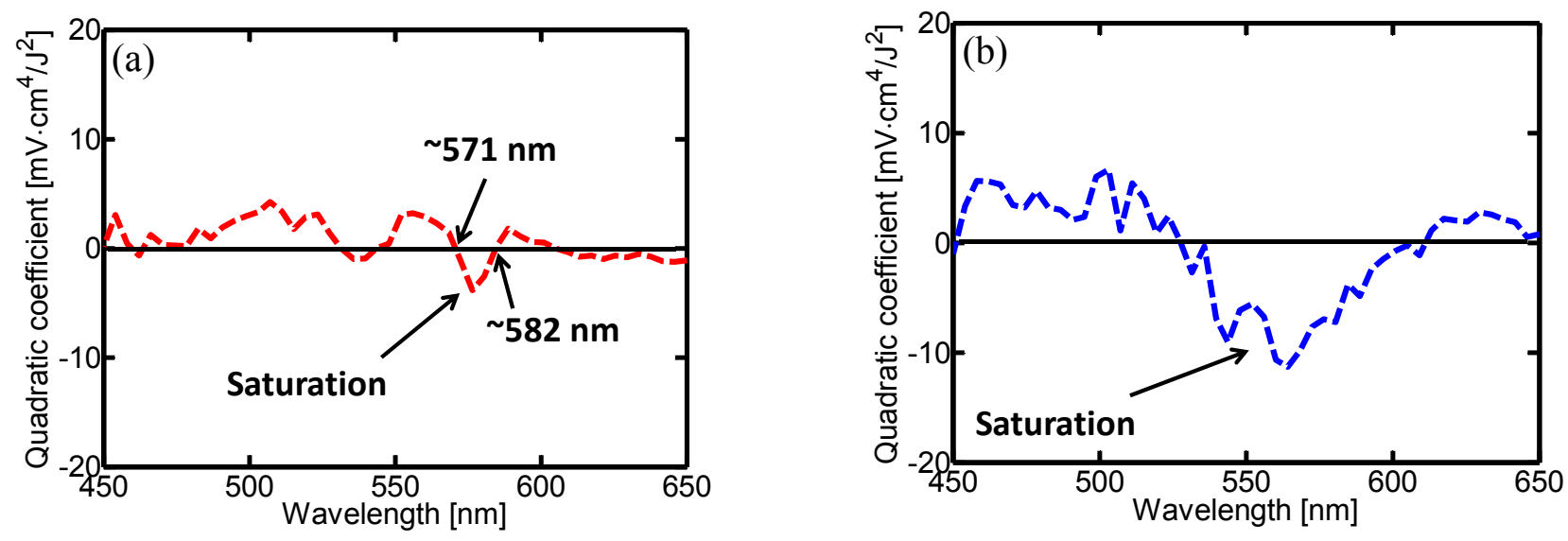

Figure 6: The quadratic coefficient as a function of the wavelength in (a) oxy-hemoglobin and (b) deoxyhemoglobin.

\section{CONCLUSSIONS}

We showed that at high intensity the PA signal generated from oxy- and deoxy-hemoglobin exhibits non-linear effects that are wavelength dependent. The dominant non-linear effect near the absorption peaks is saturation. However, away from the peaks, either thermal non-linearity or multi-step excitation dominates. In particular, some wavelengths were found to have less non-linearity than other wavelengths even at high intensity. Measurements made at these wavelengths are preferable for quantitative functional PAM, where linearity is a fundamental assumption. 


\section{REFERENCES}

[1] Laufer, J. G., Delpy, D., Elwell, C., and Beard, P. C., "Quantitative spatially resolved measurement of tissue chromophore concentrations using photoacoustic spectroscopy: application to the measurement of blood oxygenation and haemoglobin concentration," Phys. Med. Biol. 52, 141-168 (2007).

[2] Wang, X., Xie, X., Ku, G., and Wang, L. V., "Noninvasive imaging of hemoglobin concentration and oxygenation in the rat brain using high-resolution photoacoustic tomography," J. Biomed. Opt. 11, 024015 (2006).

[3] Zhang, H. F., Maslov, K., Stoica, G., and Wang, L. V., "Functional photoacoustic microscopy for high-resolution and noninvasive in vivo imaging," Nat. Biotechnol. 24, 848-851 (2006)

[4] Danielli, A., Yao J., Krumholz A., and Wang L. V., "Effects of calibration factors and intensity dependent nonlinearity on functional photoacoustic microscopy," Proc. SPIE 7899, 78993C (2011)

[5] Wang, L. V. and Wu H., [Biomedical Optics - Principles and Imaging], John Wiley \& Sons, Hoboken, 283-321 (2007)

[6] Danielli, A., Favazza, C. P., Maslov, K., and Wang, L. V., "Picosecond absorption relaxation measured with nanosecond laser photoacoustics," Appl. Phys. Lett. 97, 163701 (2010)

[7] Tam, A. C., and Patel, C. K. N., "Two-photon absorption spectra and cross-section measurements in liquids," Nature 280, 304-306 (1979)

[8] Sathy, P., Philip, R., Nampoori, V. P. N., and Vallabhan, C. P. G., "Photoacoustic observation of excited singlet state absorption in the laser dye Rhodamine 6G," J. Phys. D Appl. Phys. 27, 2019-2022 (1994)

[9] Calasso, I. G., Craig W., and Diebold G. J., "Photoacoustic point source," Phys. Rev. Lett., 86, 3550-3553 (2001) 